\title{
DETERMINACIÓN SIMULTÁNEA DE CATIONES MAYORITARIOS EN MUESTRAS DE AGUA RESIDUAL POR MEDIO DE CROMATOGRAFÍA DE IONES CON DETECCIÓN CONDUCTIMÉTRICA
}

\author{
Olivia ZAMORA MARTÍNEZ ${ }^{1 *}$, José Manuel MONTAÑO HILARIO², \\ Viviana Berenice GALINDO ZAVALA ${ }^{1}$, Christina SIEBE GRABACH ${ }^{1}$ y Blanca Lucía PRADO PANO ${ }^{1}$
}

${ }^{1}$ Departamento de Edafología, Instituto de Geología, Universidad Nacional Autónoma de México. Ciudad Universitaria, Ciudad de México, México, C.P. 04510

${ }^{2}$ Facultad de Química, Universidad Nacional Autónoma de México. Ciudad Universitaria, Ciudad de México, México, C.P. 04510

*Autor para correspondencia: oliviazm@geologia.unam.mx

(Recibido junio 2015; aceptado enero 2016)

Palabras clave: monitoreo ambiental, validación analítica, Valle del Mezquital, reuso agrícola

\section{RESUMEN}

Se desarrolló y validó una metodología para la determinación y cuantificación simultánea de cinco cationes empleando la técnica de cromatografía de iones (CI) con detección conductimétrica, con la cual fue posible monitorear el contenido de sodio, amonio, potasio, calcio y magnesio, en muestras de agua residual provenientes del Valle del Mezquital (Hidalgo, México), aun cuando éstas contenían altas concentraciones de sodio, principal interferente del ion amonio al utilizarse esta técnica, debido a que ambos cationes presentan tiempos de retención muy cercanos. Los niveles de concentración para la cuantificación de los cinco analitos van desde valores cercanos a una parte por millón hasta $100 \mathrm{mg} / \mathrm{L}$, con bajos límites de detección $(\leq 0.6 \mathrm{mg} / \mathrm{L})$, valores de precisión aceptables $(\%$ desviación estándar relativa $\leq 3.0)$ y porcentajes de exactitud muy cercanos al $100 \%$ (98.0 - 114.5\%), así como ausencia de otras interferencias.

Key words: environmental monitoring, analytical validation, Valle del Mezquital, agricultural reuse

\begin{abstract}
A methodology for the identification and simultaneous quantification of five cations using ion chromatography (IC) with conductivity detection was developed and validated. The method allows to monitor the content of sodium, ammonium, potassium, calcium and magnesium in wastewater samples from the Valle del Mezquital (Hidalgo, Mexico), despite that these contain high concentrations of sodium, main interfering ion for the quantification of ammonium, since both cations have very close retention times. Concentration levels achieved for quantification of the five analytes are between 1 to $100 \mathrm{mg} / \mathrm{L}$, with low detection limits $(\leq 0.6 \mathrm{mg} / \mathrm{L})$, good precision $(\%$ relative standard deviation $\leq 3.0$ ) and accuracy (between 98.0-114.5\%), as well as the absence of any interference.
\end{abstract}




\section{INTRODUCCIÓN}

El Valle del Mezquital está ubicado en el estado de Hidalgo aproximadamente a $80 \mathrm{~km}$ al norte de la Ciudad de México (Guédron et al. 2014). Es la zona más grande del país en la que se utiliza agua residual urbana para riego agrícola. El agua proviene de la Ciudad de México y su área conurbada (Alonso 2003). En la actualidad el reúso de agua residual para el riego de tierras agrícolas se está expandiendo en todo el mundo en las regiones áridas y semiáridas con la finalidad de optimizar el uso del agua (Feigin et al. 1991, Drechsel y Evans 2010). Sin embargo, no hay que perder de vista que los contaminantes y los agentes patógenos contenidos en las aguas residuales pueden poner en riesgo a los agricultores, a los cultivos y a los consumidores de los mismos, así como contaminar el agua subterránea en las zonas donde se aplica. Actualmente existen trabajos en los cuales se discuten los diversos efectos que provoca la presencia de especies de interés ambiental como son: metales pesados (Flores et al. 1992, Hernández et al. 1994 y Siebe 1994), pesticidas (Downs et al. 1999) y fármacos (Dalkmann et al. 2014), entre otras, en la zona agrícola del Valle del Mezquital.

La salinidad iónica de cuerpos de agua es a menudo determinada por cuatro cationes principales, calcio $\left(\mathrm{Ca}^{2+}\right)$, magnesio $\left(\mathrm{Mg}^{2+}\right)$, sodio $\left(\mathrm{Na}^{+}\right)$y potasio $\left(\mathrm{K}^{+}\right)$(Wetzel 1981). Estos elementos forman parte de funciones importantes en los organismos vivos, los cationes monovalentes de sodio y potasio están implicados principalmente en el transporte e intercambio iónico; el calcio actúa de distintas formas sobre el crecimiento y la dinámica de las poblaciones florísticas y faunísticas de las aguas dulces, y el magnesio está presente en todas las plantas con clorofila (Wetzel 1981). Por otro lado, el agua residual contiene nutrientes como el nitrógeno (N), necesario para el desarrollo de los cultivos. Sin embargo, las cantidades de $\mathrm{N}$ aportadas con el riego pueden rebasar las cantidades necesarias para el desarrollo de las plantas y sus excedentes pueden deteriorar la calidad del aire y del agua subterránea. Por ejemplo, el excedente de $\mathrm{N}$ puede desnitrificarse o volatilizarse aumentando las emisiones de gases con efecto invernadero $\left(\mathrm{N}_{2} \mathrm{O}\right.$ y $\left.\mathrm{NH}_{3}\right)$ en la atmósfera, o bien oxidarse a nitrato y contaminar el agua subterránea por lixiviación. Una de las formas de monitoreo del $\mathrm{N}$ en el agua y la solución del suelo es la cuantificación de amonio $\left(\mathrm{NH}_{4}{ }^{+}\right)$, que dadas las condiciones anóxicas que se presentan en aguas con altas cargas de materia orgánica, es la especie inorgánica de $\mathrm{N}$ dominante.
El agua residual también contiene muchas otras especies disueltas, algunas de las cuales se pueden encontrar en concentraciones muy altas con respecto a las de $\mathrm{NH}_{4}^{+}$, como lo es el contenido del ion sodio. Es por ello que para la determinación de amonio se deben desarrollar metodologías que presenten bajos límites de detección, ausencia de interferencias, intervalos dinámicos de concentración apropiados y una mínima preparación de la muestra. Lo anterior, debido a que las que existen, como es el caso de la determinación colorimétrica de amonio mediante su reacción con nitroprusiato en un medio complejante alcalino (Searle 1975) y las determinaciones de cromatografía de iones típicas (Eith et al. 2007), no cumplen con varias de esas características deseables. Mientras que para las determinaciones de $\mathrm{Na}^{+}, \mathrm{K}^{+}, \mathrm{Ca}^{2+}$ y $\mathrm{Mg}^{2+}$, los análisis suelen realizarse comúnmente por medio de la técnica de flamometría, que aunque es de bajo costo, presenta el principal inconveniente de manejar intervalos dinámicos de concentración muy cortos, por lo que siempre es necesario realizar la dilución de la muestras, en muchos casos con un factor muy alto.

Debido a la diversidad de las especies que puede contener un tipo de agua, en la actualidad se ha visto la necesidad de contar con numerosos métodos de preparación y análisis de muestras que permitan el monitoreo de estas especies. Una de las técnicas utilizadas es la cromatografía de líquidos de alta resolución (HPLC), que permite separar y cuantificar analitos de interés en matrices y mezclas más o menos complejas de manera simultánea (Neue 1997). Dentro de la clasificación de las técnicas de HPLC, se encuentra la cromatografía de iones o cromatografía iónica (CI), la cual es utilizada para la separación y determinación de solutos iónicos presentes generalmente en agua (Levin 1997). El detector más frecuentemente usado en CI es el de conductividad, que se basa en la propiedad que tienen los electrolitos de conducir la corriente eléctrica (Rouessac y Rouessac 2003). Un detector de conductividad tiene muchas de las propiedades de un detector ideal, es sensible, es universal para especies cargadas y responde de forma predecible a los cambios de concentración (Levin 1997).

Debido a esto el objetivo de este trabajo fue el desarrollo y la validación de una metodología que permita la determinación simultánea de sodio, amonio, potasio, calcio y magnesio en muestras de agua residual por medio de cromatografía de iones, con una mínima preparación de muestra, que garantice resultados confiables y que permita además la adecuada cuantificación del ion amonio en muestras que presentan altos contenidos de sodio. 


\section{MATERIALES Y MÉTODOS}

Las determinaciones se realizaron con un cromatógrafo de líquidos constituido de una bomba binaria Waters ${ }^{\circledR}$ modelo 1525 , un automuestreador Waters ${ }^{\circledR}$ modelo 717 plus y un detector de conductividad Waters ${ }^{\circledR}$ modelo 432 . Se utilizó como fase estacionaria una columna metrosep C6 de 250x4.0 mm de Metrohm ${ }^{\circledR}$, empacada con partículas de diámetro de $5 \mu \mathrm{m}$ y, como fase móvil, una mezcla de $\mathrm{HNO}_{3} \mathrm{y}$ ácido dipicolínico de igual concentración $(1.7 \mathrm{mM}$, Sigma-Aldrich), en modo isocrático a un flujo de 0.9 $\mathrm{mL} / \mathrm{min}$. Las disoluciones patrón fueron preparadas por dilución de un estándar de referencia trazable al NIST (IC-4 High-Purity Standards), utilizando agua ultra pura tipo I como disolvente (MilliQ). Con estas características cromatográficas de análisis se determinaron los parámetros típicos de una validación analítica: intervalo lineal, exactitud, precisión, límites de detección y de cuantificación y recuperación para sodio, amonio, potasio, calcio y magnesio de acuerdo con lo siguiente:

a) Intervalo lineal y de trabajo. Con este fin se prepararon estándares de concentración de los cinco analitos comprendidos entre 0.05 y 100 ppm en agua MilliQ. El intervalo mencionado anteriormente se seleccionó con base en dos criterios, la repetibilidad de la respuesta del detector y a su respectiva desviación estándar.

b). Precisión. Se determinó para tres niveles de concentración $(1,25$ y $75 \mathrm{mg} / \mathrm{L})$ de cada analito. Cada concentración fue preparada por quintuplicado de manera independiente y analizada también por quintuplicado. La evaluación de este parámetro de calidad se realizó mediante el cálculo del porcentaje de desviación estándar relativa (\% DER).

c) Exactitud. El parámetro de exactitud fue calculado a partir de la inyección de cinco disoluciones independientes que contenían $\mathrm{Na}^{+}, \mathrm{NH}_{4}^{+}, \mathrm{K}^{+}, \mathrm{Ca}^{2+}$ y $\mathrm{Mg}^{2+}$ para tres niveles de concentración $(5,50$ y 100 $\mathrm{mg} / \mathrm{L}$ ). Con los valores de área determinados y los parámetros de regresión calculados de las curvas de calibración, se determinó la concentración de cada estándar y se comparó con el valor conocido.

d) Límites de detección (LD) y de cuantificación (LC). Estos dos parámetros fueron determinados utilizando la señal analítica que se obtiene al analizar disoluciones de una muy baja concentración de cada uno de los cationes de trabajo. Para su obtención se realizaron siete inyecciones de disoluciones de concentración conocida de cada uno de los analitos $(0.05 \mathrm{mg} / \mathrm{L}$ para sodio y amonio, $0.1 \mathrm{mg} / \mathrm{L}$ para potasio $\mathrm{y}$
$0.5 \mathrm{mg} / \mathrm{L}$ para calcio y magnesio). Con los valores de área determinados se calcularon la media $(\bar{X})$ y la desviación estándar (s) de cada especie de estudio. El límite de detección se reporta de acuerdo con lo establecido por Miller y Miller (2002) con la ecuación:

$\mathrm{LD}=\bar{X}+3 \mathrm{~s}$

Para el límite de cuantificación se utilizó la ecuación también establecida por Miller y Miller (2002):

$\mathrm{LC}=\bar{X}+10 \mathrm{~s}$

Posteriormente, con la ecuación de la curva de calibración se obtuvieron los valores de los límites de detección y de cuantificación en unidades de concentración.

e) Recuperación. Este parámetro se determinó por medio de la adición del volumen necesario de un estándar que contenía $100 \mathrm{mg} / \mathrm{L}$ de cada catión de estudio, la cual provocará un incremento en la concentración de sodio, amonio, potasio, calcio y magnesio de $10 \mathrm{mg} / \mathrm{L}$ en trece muestras de agua residual provenientes del Valle del Mezquital. Con los valores de área determinados de las muestras fortificadas y sin fortificar, y los parámetros de regresión calculados de las curvas de calibración, se determinaron las concentraciones de $\mathrm{Na}^{+}, \mathrm{NH}_{4}{ }^{+}, \mathrm{K}^{+}$, $\mathrm{Ca}^{2+} \mathrm{y} \mathrm{Mg}^{2+}$ resultantes del paso de fortificación.

f) Evaluación de interferencias (sodio-amonio). En el caso de la determinación de amonio y debido a la naturaleza de las muestras de trabajo, fue necesario realizar un estudio de selectividad más específico. Para esto se seleccionaron muestras de agua residual que no contuvieran amonio, se analizaron con la metodología desarrollada y posteriormente fueron fortificadas con amonio y vueltas a analizar. Como segundo paso se realizó la evaluación de la máxima relación sodio-amonio presente en muestras de agua que el sistema cromatográfico de trabajo pudiera analizar sin presentar el traslapamiento de las señales de estos dos cationes. Se evaluaron cuatro relaciones sodio-amonio, 1:1, 10:1, 100:1 y 1000:1. Finalmente, ya que el principal interferente en la determinación del ion amonio por medio de cromatografía de iones en las muestras de agua residual de estudio es el sodio, se decidió verificar la concentración de $\mathrm{NH}_{4}{ }^{+}$utilizando una técnica alternativa para poder comparar los resultados obtenidos por ambas metodologías. Para ello se cuantificó el contenido de amonio en 30 muestras de agua residual con dos metodologías, la propuesta en el presente trabajo y una de rutina establecida en otro laboratorio. 
Esta consiste en una determinación colorimétrica a $660 \mathrm{~nm}$ de un derivado químico del ion amonio, producido en medio básico por medio de una reacción con nitroprusiato de sodio utilizando un analizador automático (Technicon AutoAnalyzer II). Los resultados obtenidos fueron comparados y evaluados mediante las pruebas $t$ de Student, F de Fisher y análisis de varianza. Las concentraciones medias obtenidas con cada metodología $(n=3)$, fueron comparadas a un de nivel de confianza del $95 \%$. Para los tres casos se utilizó el programa Statgraphics XVII®.

Las muestras de agua residual utilizadas para realizar los experimentos de fortificación fueron tomadas en diferentes sitios de muestreo en la localidad de Tlahuelilpan, ubicada en el centro del Valle del Mezquital. Dichas muestras fueron filtradas in situ utilizando membranas de nailon de $0.45 \mu \mathrm{m}$ (Millipore) y conservadas en hieleras para garantizar su estabilidad hasta su llegada al laboratorio, en donde fueron colocadas bajo refrigeración a $4{ }^{\circ} \mathrm{C}$.

\section{RESULTADOS Y DISCUSIÓN}

\section{Condiciones cromatográficas}

Para contar con un sistema adecuado de cuantificación de amonio en las muestras de agua residual provenientes del Valle del Mezquital, se evaluaron más de una fase estacionaria y diferentes composiciones de fase móvil con base en lo reportado por Eith et al. (2007). Estos autores mencionan que es posible hacer la determinación simultánea no solamente de $\mathrm{Na}^{+} \mathrm{y} \mathrm{NH}_{4}^{+}$, sino también de $\mathrm{K}^{+}, \mathrm{Ca}^{2+}$ y $\mathrm{Mg}^{2+}$ en niveles de concentración de miligramos por litro, pero no dan información acerca de las rela- ciones sodio-amonio que se pueden manejar en esas condiciones para evitar solapamiento de señales. Sin embargo, al analizar las muestras de estudio con estos protocolos, se presentó la coelución de los iones sodio y amonio (traslapamiento de señales), debido a que el contenido presente de $\mathrm{Na}^{+}$ resulta ser muy alto respecto al de $\mathrm{NH}_{4}{ }^{+}$. Además, dado que los tiempos de retención entre estos dos analitos difieren (en el mejor de los casos en 1.3 min), no fue posible su completa separación. Cabe mencionar que se intentó mejorar dicha separación por medio de la combinación de dos factores que afectan directamente a la retención cromatográfica: la disminución de la fuerza de la fase móvil y el aumento de la longitud de la fase estacionaria. Los resultados obtenidos mostraron un ligero incremento en la separación de las señales pero ésta aún no fue completa. En este caso particular tampoco fue factible la dilución de la muestra, ya que los factores de dilución necesarios provocaron que la concentración del amonio no fuera siquiera detectable. Finalmente, al utilizar una columna que presenta una alta capacidad de carga y una mejor eficiencia (Metrosep C6 de $250 \times 4.0 \mathrm{~mm}$ ) se logró una excelente separación de los analitos comprometidos (sodio y amonio) y su adecuada cuantificación sin la necesidad de realizar ningún paso de dilución en las muestras del agua residual de estudio.

En la figura 1 se presenta el cromatograma obtenido cuando un multiestándar de $10 \mathrm{mg} / \mathrm{L}$ de los cinco cationes es analizado. Como se puede observar es posible determinar y cuantificar de manera simultánea a los cinco cationes en un tiempo total de corrida de $46 \mathrm{~min}$, sin la coelución de los iones sodio y amonio. En el cuadro I se presentan los tiempos de retención y los factores de capacidad de cada una de las especies de estudio, así como

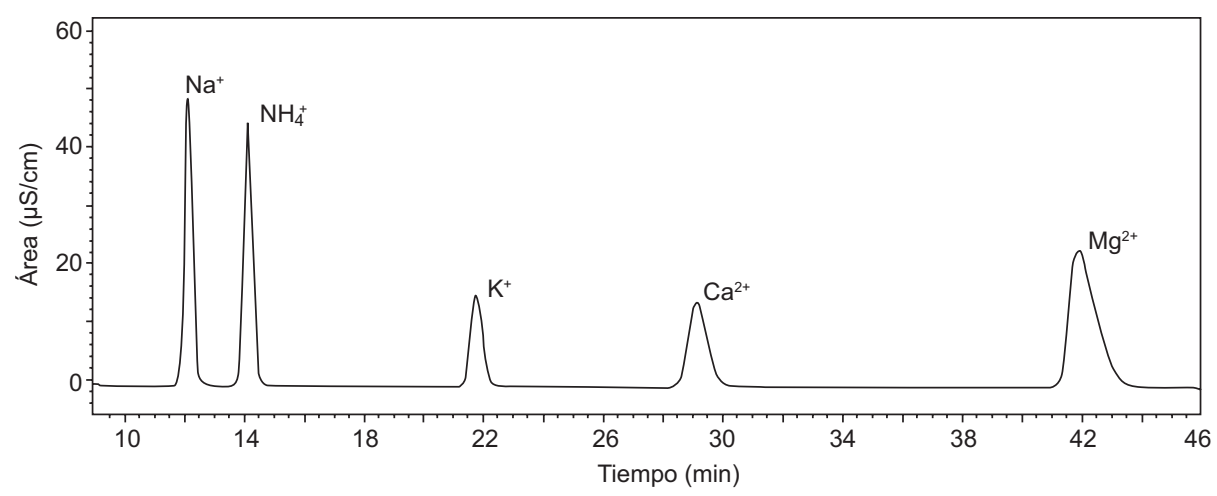

Fig. 1. Cromatograma obtenido para una disolución que contiene $\mathrm{Na}^{+}, \mathrm{NH}_{4}^{+}, \mathrm{K}^{+}, \mathrm{Ca}^{2+}$ y $\mathrm{Mg}^{2+}$ en concentración igual a $10 \mathrm{mg} / \mathrm{L}$. Fase estacionaria: columna catiónica C6 de 250x4.0 mm, fase móvil: mezcla $\mathrm{HNO}_{3}$ y ácido dipicolínico de igual concentración (1.7 mM) 
CUADRO I. PARÁMETROS CROMATOGRÁFICOS DEL SISTEMA DE ANÁLISIS UTILIZADO

\begin{tabular}{lccccc}
\hline Analito & $\begin{array}{c}\text { Tiempo de } \\
\text { retención (min) }\end{array}$ & $\begin{array}{c}\text { Tiempo de retención } \\
\text { corregido (min) }\end{array}$ & $\begin{array}{c}\text { Factor de } \\
\text { capacidad }\end{array}$ & Selectividad & Resolución \\
\hline $\mathrm{Na}^{+}$ & 12.0 & 9.7 & 4.2 & 1.2 & 2.5 \\
$\mathrm{NH}_{4}^{+}$ & 14.0 & 11.7 & 5.1 & & \\
\hline $\mathrm{K}^{+}$ & 21.8 & 19.5 & 8.5 & \\
$\mathrm{Ca}^{2+}$ & 29.0 & 26.7 & 11.6 & \\
$\mathrm{Mg}^{2+}$ & 42.0 & 39.7 & 17.3 & & \\
\hline
\end{tabular}

los valores de selectividad y de resolución para los iones sodio y amonio, con los cuales es posible decir que se logra una adecuada separación de estas especies.

\section{Validación de la metodología propuesta Intervalos lineales}

Se determinaron los intervalos lineales para cada uno de los cationes de estudio. En el cuadro II se resumen los resultados de los análisis de regresión obtenidos al tratar las curvas de calibración de los cinco analitos de interés mostrándose, para todos ellos, intervalos lineales de concentración grandes (de hasta casi tres niveles de magnitud) y valores aceptables de coeficientes de correlación ( $>0.998)$. Cabe mencionar que para cada uno de los cinco analitos de estudio se llevó a cabo la prueba no paramétrica de rachas con los residuales de las respectivas parejas de datos que constituyen las curvas de calibración, encontrándose en todos los casos que los residuos de éstos son aleatorios, por lo que es posible decir que los datos se ajustan a una recta. Para todos los analitos se observan valores de pendiente $>0$, lo cual se confirmó al realizar la respectiva prueba de hipótesis, en donde se estableció como hipótesis nula, $\mathrm{H}_{0}$, que el valor de la pendiente $(\mathrm{m})$ de cada curva de calibración es igual a cero y como hipótesis alterna, $\mathrm{H}_{1}$, que el valor de la pendiente es mayor que $0 . \mathrm{Al}$ realizar la prueba de t para cada una de las curvas de calibración, al $95 \%$ de confianza, los valores de $t_{\text {calculados }}$ fueron mayores que los valores de $t_{\text {tablas, }}$ por lo que en todos los casos la hipótesis nula fue rechazada, es decir, $m>0$.

En la figura 2 se presentan las curvas de calibración para cada uno de los cationes determinados. El analito más sensible para su cuantificación por medio de esta metodología resultó ser el $\mathrm{Mg}^{2+}$, ya que presenta el valor más alto de pendiente, mientras que el sodio y el ion amonio presentan sensibilidades muy parecidas.

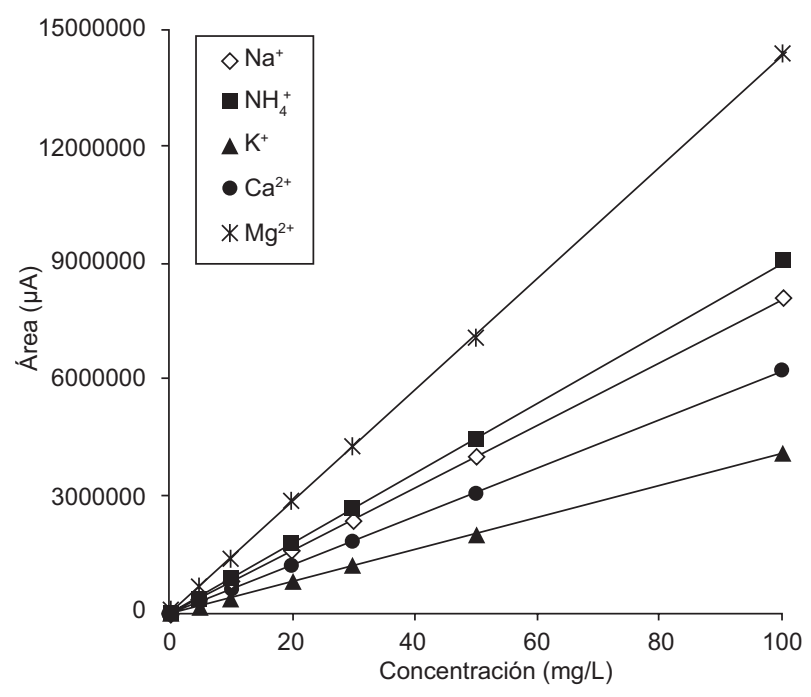

Fig. 2. Curvas de calibración para cada uno de los cationes determinados $(n=9)$

CUADRO II. Resultados de los análisis de regresión lineal para los cinco analitos $(n=9)$

\begin{tabular}{lcccc}
\hline \multirow{4}{*}{ Analito } & \multicolumn{3}{c}{ Parámetro } \\
\cline { 2 - 5 } & $\begin{array}{c}\text { Intervalo lineal de } \\
\text { concentración }(\mathrm{mg} / \mathrm{L})\end{array}$ & $\begin{array}{c}\text { Ordenada al } \\
\text { origen }\end{array}$ & Pendiente & $\begin{array}{c}\text { Coeficiente de } \\
\text { correlación }\end{array}$ \\
\hline $\mathrm{Na}^{+}$ & $0.3-100$ & $4000 \pm 26000$ & $80500 \pm 660$ & 0.9998 \\
$\mathrm{NH}_{4}^{+}$ & $0.2-100$ & $-22000 \pm 31000$ & $91140 \pm 790$ & 0.9998 \\
$\mathrm{~K}^{+}$ & $0.5-100$ & $-5000 \pm 21000$ & $41680 \pm 530$ & 0.9995 \\
$\mathrm{Ca}^{2+}$ & $1-100$ & $2000 \pm 54000$ & $62200 \pm 1300$ & 0.9989 \\
$\mathrm{Mg}^{2+}$ & $1-100$ & $-44000 \pm 69000$ & $146400 \pm 1800$ & 0.9996 \\
\hline
\end{tabular}




\section{Exactitud y precisión}

Se presentan los resultados de precisión, parámetro evaluado como desviación estándar relativa (Cuadro III), así como la exactitud (Cuadro IV). En el caso de la precisión se encontraron para los cinco cationes en los tres niveles de concentración evaluados (1, 25 y $75 \mathrm{mg} / \mathrm{L})$, valores de $\%$ DER $<3$, por lo que de acuerdo con Taverniers et al. (2004), es posible decir que se cuenta con un sistema de medición preciso. En cuanto a la exactitud, los valores determinados para los cinco cationes en los tres niveles de concentración fueron muy cercanos al $100 \%$ sin exceder una diferencia mayor al $15 \%$ por lo que también se puede afirmar que se cuenta con un sistema exacto.

CUADRO III. EVALUACIÓN DE LA PRECISIÓN DEL SISTEMA DESARROLLADO PARA TRES NIVELES DE CONCENTRACIÓN $(n=5)$

\begin{tabular}{cccccc}
\hline \multirow{2}{*}{$\begin{array}{c}\text { Concentración } \\
(\mathrm{mg} / \mathrm{L})\end{array}$} & \multicolumn{5}{c}{ \% desviación estándar relativa } \\
\cline { 2 - 6 } & $\mathrm{Na}^{+}$ & $\mathrm{NH}_{4}^{+}$ & $\mathrm{K}^{+}$ & $\mathrm{Ca}^{2+}$ & $\mathrm{Mg}^{2+}$ \\
\hline 1 & 3.0 & 2.9 & 1.1 & 2.4 & 2.2 \\
25 & 1.6 & 1.7 & 1.9 & 1.5 & 1.3 \\
75 & 0.2 & 0.3 & 0.4 & 0.6 & 0.3 \\
\hline
\end{tabular}

CUADRO IV. EVALUACIÓN DE LA EXACTITUD DEL SISTEMA DESARROLLADO PARA TRES NIVELES DE CONCENTRACIÓN $(\mathrm{n}=5)$.

\begin{tabular}{crrrrr}
\hline \multirow{2}{*}{$\begin{array}{c}\text { Concentración } \\
(\mathrm{mg} / \mathrm{L})\end{array}$} & \multicolumn{5}{c}{ \% exactitud } \\
\cline { 2 - 6 } & \multicolumn{1}{c}{$\mathrm{Na}^{+}$} & $\mathrm{NH}_{4}{ }^{+}$ & \multicolumn{1}{c}{$\mathrm{K}^{+}$} & $\mathrm{Ca}^{2+}$ & $\mathrm{Mg}^{2+}$ \\
\hline 5 & 98.0 & 98.6 & 108.0 & 104.2 & 101.7 \\
50 & 110.3 & 103.6 & 111.0 & 110.2 & 104.4 \\
100 & 114.5 & 106.6 & 114.1 & 114.0 & 108.4 \\
\hline
\end{tabular}

\section{Límites de detección y cuantificación}

En el cuadro $\mathbf{V}$ se resumen los valores obtenidos, reportándose los límites de detección (LD) y de cuantificación (LC) de acuerdo con lo establecido por Miller y Miller (2002). En todos los casos, los valores de los $\mathrm{LD}$ son menores que $1 \mathrm{mg} / \mathrm{L}$ y el valor del LC determinado para cada uno de los cationes se encuentra muy cercano a la concentración más baja que forma parte del intervalo lineal de concentración de cada analito. Estos valores indican que el método desarrollado puede ser aplicado para realizar la caracterización de muestras de aguas residuales, ya que los contenidos de sodio, amonio, potasio, calcio y magnesio en este tipo de muestras son, en la mayoría de los casos $>1 \mathrm{mg} / \mathrm{L}$.
CUADRO V. VALORES DE LÍMITES DE DETECCIÓN Y DE CUANTIFICACIÓN PARA LOS CINCO ANALITOS DE INTERÉS $(\mathrm{n}=7)$

\begin{tabular}{ccc}
\hline \multirow{2}{*}{ Analito } & \multicolumn{2}{c}{ Parámetro } \\
\cline { 2 - 3 } & $\begin{array}{c}\text { Límite de } \\
\text { detección } \mathrm{mg} / \mathrm{L}\end{array}$ & $\begin{array}{c}\text { Límite de } \\
\text { cuantificación } \mathrm{mg} / \mathrm{L}\end{array}$ \\
\hline $\mathrm{Na}^{+}$ & 0.06 & 0.27 \\
$\mathrm{NH}_{4}^{+}$ & 0.12 & 0.13 \\
$\mathrm{~K}^{+}$ & 0.24 & 0.56 \\
$\mathrm{Ca}^{2+}$ & 0.5 & 0.9 \\
$\mathrm{Mg}^{2+}$ & 0.6 & 1.0 \\
\hline
\end{tabular}

\section{Recuperaciones}

Se determinaron los porcentajes de recuperación en trece muestras de agua residual fortificadas con concentraciones conocidas de $\mathrm{Na}^{+}, \mathrm{NH}_{4}{ }^{+}, \mathrm{K}^{+}, \mathrm{Ca}^{2+} \mathrm{y} \mathrm{Mg}^{2+}$ (a un nivel de concentración, $10 \mathrm{mg} / \mathrm{L}$ ), los valores obtenidos se muestran en el cuadro VI. Los resultados presentan en todos los casos recobros comprendidos entre el 80 y $110 \%$ por lo que de acuerdo con Taverniers et al. (2004), son analíticamente aceptables. La obtención de porcentajes de recuperación muy cercanos a1 $100 \%$ indica que la metodología propuesta no presenta interferencias en la determinación de los cinco cationes de estudio, por lo que los resultados generados son confiables. Sin embargo, debido al tipo de muestras (aguas residuales), es necesario realizar una evaluación más detallada sobre la cuantificación del ion amonio, ya que la presencia de una alta concentración de sodio puede generar resultados erróneos acerca del contenido de $\mathrm{NH}_{4}{ }^{+}$.

CUADRO VI. PORCENTAJES DE RECUPERACIÓN OBTENIDOS AL FORTICAR TRECE MUESTRAS DE AGUA RESIDUAL PROVENIENTES DEL VALLE DEL MEZQUITAL CON $10 \mathrm{mg} / \mathrm{L}$ DE LOS CATIONES Na $\mathrm{NH}_{4}^{+}, \mathrm{K}^{+}, \mathrm{Ca}^{2+} \mathrm{Y} \mathrm{Mg}^{2+}$

\begin{tabular}{lrrrrr}
\hline \multirow{2}{*}{ Muestra } & \multicolumn{5}{c}{ \% recuperación $(\mathrm{n}=3)$} \\
\cline { 2 - 6 } & \multicolumn{1}{c}{$\mathrm{Na}^{+}$} & $\mathrm{NH}_{4}^{+}$ & \multicolumn{1}{c}{$\mathrm{K}^{+}$} & \multicolumn{1}{c}{$\mathrm{Ca}^{2+}$} & $\mathrm{Mg}^{2+}$ \\
\hline M1 & 89.7 & 80.5 & 93.0 & 95.2 & 109.2 \\
M2 & 88.5 & 101.9 & 96.7 & 96.0 & 101.4 \\
M3 & 87.5 & 80.8 & 95.4 & 99.8 & 108.5 \\
M4 & 97.8 & 84.4 & 90.6 & 83.0 & 92.7 \\
M5 & 89.1 & 82.5 & 88.5 & 88.9 & 94.3 \\
M6 & 99.4 & 81.6 & 86.9 & 90.1 & 96.2 \\
M7 & 103.4 & 80.4 & 86.5 & 93.3 & 98.3 \\
M8 & 94.9 & 86.2 & 87.4 & 85.3 & 95.2 \\
M9 & 93.8 & 83.0 & 97.2 & 100.7 & 103.5 \\
M10 & 90.5 & 83.2 & 95.2 & 101.9 & 106.9 \\
M11 & 81.1 & 84.4 & 81.5 & 87.8 & 92.8 \\
M12 & 110.5 & 86.6 & 88.1 & 86.2 & 94.3 \\
M13 & 100.4 & 82.5 & 82.4 & 87.2 & 95.9 \\
\hline
\end{tabular}




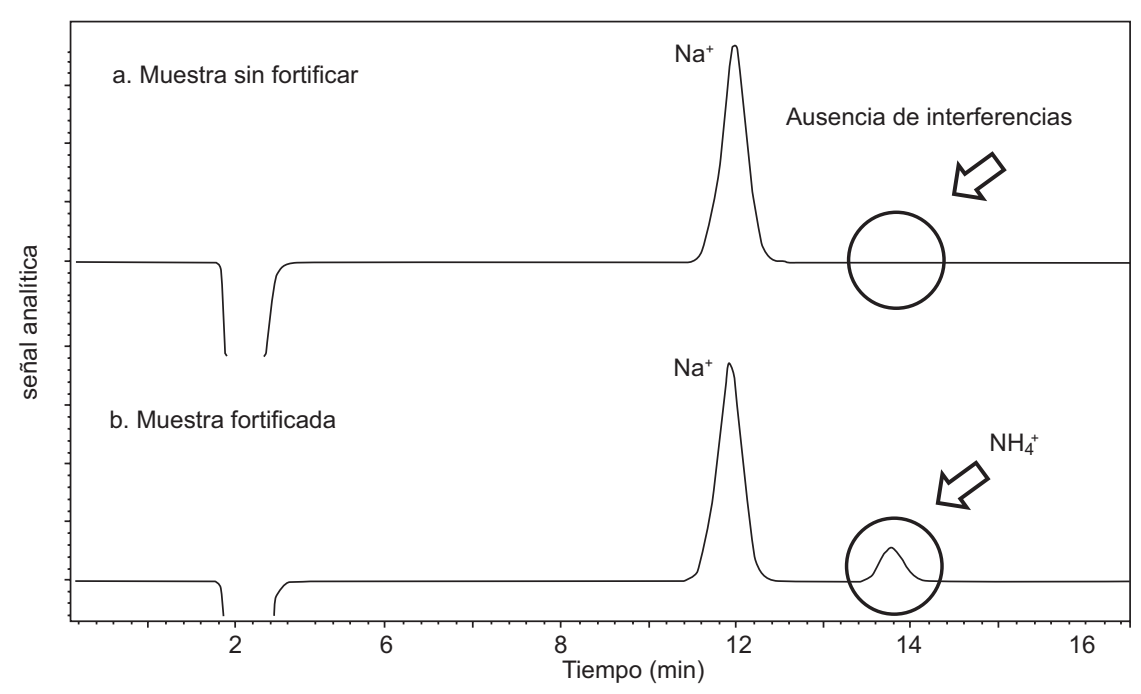

Fig. 3. Evaluación de la selectividad cromatográfica del sistema de análisis para el ion amonio

\section{Evaluación de interferencias (sodio-amonio) Selectividad}

En el caso de la cuantificación del ion amonio fue posible evaluar la selectividad del sistema cromatográfico desarrollado, ya que se contó con muestras reales que no contenían este analito. En la figura 3, en el cromatograma a, se presenta el resultado obtenido al analizar una muestra de agua residual proveniente del Valle del Mezquital que se sabe no contiene amonio, mientras que en el cromatograma $b$, se presenta el resultado obtenido al realizar el análisis de un fortificado de amonio en la misma muestra. Como se puede comprobar, en la muestra sin fortificar no se observa una señal en el tiempo correspondiente al ion amonio, mientras que en la fortificada sí existe. Considerando lo anterior es posible decir que el método de análisis utilizado en las muestras de agua residuales de esta investigación está libre de interferencias. Debido a que los iones sodio y amonio presentan tiempos de retención muy cercanos y porque que las muestras de estudio tuvieron concentraciones elevadas del primero respecto al segundo, es posible que exista un traslapamiento de señales. Por lo anterior es necesario conocer la máxima relación sodio-amonio que puede tolerar la metodología propuesta en el este trabajo para así generar resultados adecuados.

\section{Relaciones de estudio $\mathrm{Na}^{+}-\mathrm{NH}_{4}{ }^{+}$}

Debido a que no se conoce la relación máxima sodio-amonio para evitar el traslapamiento de las señales de estos dos iones, se evaluaron las cuatro relaciones mostradas en la figura 4. Como se puede observar, aun cuando exista una concentración de

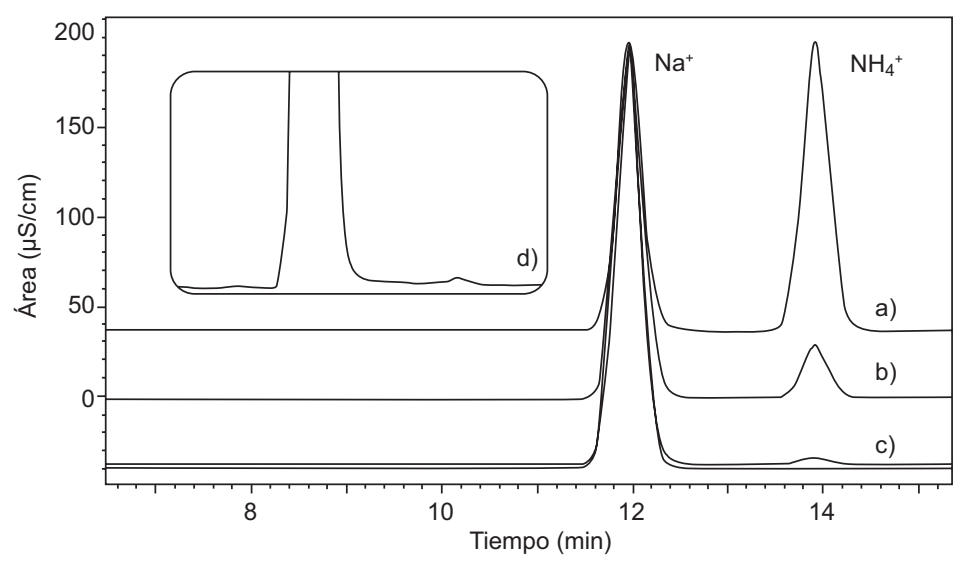

Fig. 4. Relaciones $\mathrm{Na}^{+}-\mathrm{NH}_{4}{ }^{+}$evaluadas: a) $1: 1$, b) 10:1, c) 100:1 y d) 1000:1 
mil veces más sodio con respecto a la de amonio, se logra una adecuada separación de los analitos y será posible su cuantificación siempre y cuando la concentración de ambos pueda ser determinada, es decir, considerando los respectivos intervalos lineales de trabajo y los valores del LD de los analitos de estudio.

\section{Intercomparación de metodologías}

Al comparar los resultados obtenidos por dos métodos de análisis diferentes: el desarrollado en el presente trabajo y la prueba colorimétrica de Searle (1975) para la cuantificación de $\mathrm{NH}_{4}{ }^{+}$en 30 muestras de agua residual provenientes del Valle del Mezquital, se encontró que los resultados generados por ambos sistemas de análisis son estadísticamente equivalentes, ya que no existe diferencia significativa (al 95\% de confianza), entre las medias y los valores de desviación estándar de las concentraciones reportadas por ambos métodos. Por lo anterior la cromatografía de iones es una excelente alternativa para el análisis de amonio en las muestras de agua residual del sitio de estudio.

\section{CONCLUSIONES}

Mediante el uso de la cromatografía de iones se implementó una metodología para la determinación simultánea de cinco cationes: sodio, amonio, potasio, calcio y magnesio en muestras de aguas residuales provenientes del Valle del Mezquital. Con esta metodología es posible determinar concentraciones del orden de $\mathrm{mg} / \mathrm{L}$ de todos los cationes de estudio, aun cuando la concentración de sodio, el principal interferente del ion amonio, sea hasta 1000 veces más elevada. Asimismo, se mostró que el método presenta valores de precisión y exactitud analíticamente aceptables, bajos límites de detección y de cuantificación y ausencia de otras interferencias debidas a otros componentes de las muestras de trabajo. La metodología propuesta puede ser usada como una herramienta para el monitoreo el agua de riego y de muestras de solución de suelo en sistemas agrícolas que reúsan agua residual.

\section{AGRADECIMIENTOS}

Los autores agradecen a Enrique Solís Villalpando del Instituto de Ecología de la Universidad Nacional Autónoma de México, por los análisis colorimétricos de amonio. A Lucero Hernández Martínez y a los alumnos del curso de monitoreo ambiental quienes colectaron las muestras en campo y a la Dirección General de Asuntos del Personal Académico (DGAPA) por financiar su desarrollo a través de los proyectos PE102113 y PE101316 del Programa de Apoyo a Proyectos para la Innovación y Mejoramiento de la Enseñanza (PAPIME).

\section{REFERENCIAS}

Alonso M. E. (2003). Biología: un enfoque integrador. $2^{\text {a }}$ ed., McGraw Hill, Ciudad de México, México, 279 pp.

Dalkmann P., Siebe C., Amelung W. y Siemens J. (2014). Does long-term irrigation with wastewater accelerate the dissipation of pharmaceuticals in soil? Environ. Sci. Technol. 48, 4963-4970. DOI: 10.1021/es501180x.

Downs T. J., Cifuentes E. y Suffet I. M. (1999). Risk screening for exposure to groundwater pollution in a wastewater irrigation District of the Mexico City region. Environ. Health Persp. 107, 553-561.

Drechsel P. y Evans A. E. V. (2010). Wastewater use in irrigated agriculture. Irrig. Drainage Syst. 24, 1-3.

Eith C., Kolb M., Rumi C. M., Seubert A. y Viehweger K. H. (2007). Practical ion chromatography, an Introduction. $2^{a}$ ed., Metrohm. Herisau, Suiza, 142 pp.

Feigin A., Ravina I. y Shalhevet J. (1991). Irrigation with treated sewage effluent. Management for environmental protection. Advanced series in agricultural sciences, Vol. 17, Springer. Nueva York, EUA, 446 pp.

Flores L., Hernández G., Alcalá R. y Maples M. (1992). Total content of cadmium, copper, manganese and zinc in agricultural soils irrigated with wastewater from Hidalgo, Mexico. Rev. Int. Contam. Ambie. 8, 37-46.

Guédron S., Duwig C., Prado B. L., Point D., Flores M.G. y Siebe C. (2014). (Methyl)mercury, arsenic, and lead contamination of the world's largest wastewater irrigation system: the Mezquital Valley (Hidalgo state-Mexico). Water Air Soil Poll. 225, 3-21. DOI: 10.1007/s11270-014-2045-3.

Hernández G., Flores L., Maples M., Solorio J. G. y Alcalá J. R. (1994). Riesgo de acumulación de Cd, Pb, Cr y Co en tres series de suelos del DR03, Estado de Hidalgo, México. Rev. Int. Contam. Ambie. 11, 53-61.

Levin S. (1997). Analysis of ions using high performance liquid chromatography: ion-chromatography, Medtechnica-analytical department, [en línea]. http://www.forumsci.co.il/HPLC/ion_chrm.html 16/02/2015.

Miller J.N. y Miller J.C. (2002). Estadística y quimiometría para química analítica. $4^{\mathrm{a}}$. ed., Prentice Hall. Madrid, España, 278 pp. 
Neue U. D. (1997). HPLC Columns theory, Technology, and Practice. Wiley-Vch, Nueva York, EUA, 405 pp.

Rodríguez R. (2000). Aportaciones al conocimiento del estado medioambiental de hidrosistemas de interés internacional situados en Castilla-La Mancha. Tesis Doctoral, Universidad de Castilla-La Mancha, Ciudad Real, España, 575 pp.

Roldán G. y Ramírez J.J. (2008). Fundamentos de limnología neotropical. Colección Ciencia y Tecnología, $2^{a}$ ed., Universidad de Antioquia, Medellín, Colombia, $440 \mathrm{pp}$.

Rouessac F. y Rouessac A. (2003). Análisis químico. Métodos y técnicas instrumentales modernas. McGraw Hill, Madrid, España, 441 pp.
Searle P. L. (1975). Automated colorimetric determination of ammonium ions in soil extracts with 'Technicon AutoAnalyzer II’ equipment. N. Z. J. Agric. Res. 18, 183-187. DOI: 10.1080/00288233.1975.10421021.

Siebe Ch. (1994). Acumulación y disponibilidad de metales pesados en suelos regados con aguas residuales en el Distrito de Riego 03, Tula, Hidalgo. Rev. Int. Contam. Ambie. 10, 15-21.

Taverniers I., De Loose M. y Van Bockstaele E. (2004). Trends in quality in the analytical laboratory. II. Analytical method validation and quality assurance. TrAC. 23, 535-552. DOI: 10.1016/j.trac.2004.04.001.

Wetzel R. G. (1981). Limnología. Ediciones Omega S. A. Barcelona, España, 679 pp. 